\title{
Resonances and thermonuclear reaction rates for charged particle collisions
}

\author{
M. Ueda* \\ Akita National College of Technology, Iijima Bunkyo-cho 1-1, Akita, 011-8511, Japan \\ A. J. Sargeant, ${ }^{\dagger}$ M. P. Pato, ${ }^{\ddagger}$ and M. S. Hussein ${ }^{\S}$ \\ Instituto de Física, Universidade de São Paulo, Caixa Postal 66318, 05315-970 São Paulo, SP, Brazil
}

(Received 19 May 2004; published 16 August 2004)

\begin{abstract}
We derive an approximate analytic expression for thermonuclear reaction rate for charged particles for the case that the cross section contains a single narrow or broad resonance described by a Breit-Wigner shape. An asymptotic series is obtained by making a Taylor series expansion which is exact at both the effective energy of the Gamow window and at the resonance energy. The resulting expression is uniformly valid as the effective energy and resonance energy coalesce or separate and as the resonance width is varied from narrow to broad. This is not true of conventional analytic approximations. We compare our uniform approximation with the exact reaction rate numerically and find it to be accurate over a large parameter range. We use our expressions to calculate the reaction rate for ${ }^{12} \mathrm{C}(p, \gamma){ }^{13} \mathrm{~N}$.
\end{abstract}

DOI: 10.1103/PhysRevC.70.025802

PACS number(s): 26.20.+f, 24.30.Gd

\section{INTRODUCTION}

The description of energy production in stars and of nucleosynthesis of the elements requires the calculation of thousands of thermonuclear reaction rates. To accompany the evolution of a star these rates must be calculated as a function of the stellar temperature and for each temperature the reactions of different species will be important as determined by folding the nuclear cross sections with the MaxwellBoltzmann distribution. Normally a combination of numerical integration and analytical techniques appropriate to the energy dependence of the cross section for each reaction is used [1-3]. While the importance of analytical techniques in numerical evaluations is diminished by increased computing power they will always be useful for understanding and checking numerical results (Ref. [4] reviews analytic techniques). Reference [1] calculated the contribution of narrow resonances in the simplest possible manner, which is to approximate the Maxwell-Boltzmann distribution by its value at the resonance energy. This approximation can be expected to be good for resonances which do not overlap significantly with the Gamow peak. Here we derive an improved formula for the inclusion of resonances in charged particle reactions which removes this restriction.

In Ref. [5] we developed an asymptotic expansion for the thermonuclear reaction rate in terms of the effective astrophysical $S$ factor $S_{\text {eff }}$ using the method of Dingle [6]. It may used in case cases where $S(E)$ can be reliably expanded as a Taylor series. Two alternative expressions for $S_{\text {eff }}$ were obtained by expanding $S(E)$ about $E=0$ and about $E=E_{0}, E_{0}$ being the effective energy of the Gamow window. From these expressions, all the approximate formulas for $S_{\text {eff }}$ com-

\footnotetext{
*Electronic address: mueda@ipc.akita-nct.ac.jp

†Electronic address: adams@ fma.if.usp.br

*Electronic address: mpato@if.usp.br

${ }^{\S}$ Electronic address: hussein@fma.if.usp.br
}

monly used [7] when $S(E)$ is slowly varying may be obtained as special cases.

The validity of the expressions derived in Ref. [5] is limited by the radii of convergence of the Taylor series expansions of $S(E)$, that is, by the location of the poles of $S(E)$. The poles may be due bound states of the composite nucleus. Illustrative is the case of ${ }^{7} \mathrm{Be}+p \rightarrow{ }^{8} \mathrm{~B}+\gamma[8]$ whose $S(E)$ has a pole at $E=-E_{B}, E_{B}=137.5 \mathrm{keV}$ being the binding energy of ${ }^{8}$ B. (Ref. [9] discusses effects other than the sub-threshold pole which can produce the low energy rise seen in the $S$-factors of several capture reactions.) There may also be poles in $S(E)$ due to resonances of the reaction pair in which case the cross section may be parametrized in the region of the resonance by the Breit-Wigner form (see Eq. (7) below) which has two poles, or the Lorentzian form [10] which has four poles. In this paper we modify the method of Ref. [5] to obtain an asymptotic expansion of the thermonuclear reaction rate for charged particles for the case that the cross section contains a single narrow or wide resonance described by a Breit-Wigner shape. Our result is uniformly valid as the resonance and Gamow peak coalesce or separate and as the resonance width is varied from narrow to broad.

Basic definitions follow in Sec. II, the limitations of conventional approximations to the reaction rate are discussed in Sec. III and the uniform approximation is derived and discussed in Sec. IV. In Sec. V we apply our formula to the reaction ${ }^{12} \mathrm{C}(p, \gamma){ }^{13} \mathrm{~N}$ which is important in the $\mathrm{CNO}$ cycle and some conclusions are drawn in Sec. VI.

\section{BASIC DEFINITIONS}

For a given reaction cross section $\sigma(E)$, the Maxwellian averaged reaction rate per particle pair is given by [11]

$$
\langle\sigma v\rangle=\left(\frac{8}{\pi \mu}\right)^{1 / 2}\left(\frac{1}{k_{B} T}\right)^{3 / 2} \int_{0}^{\infty} d E E \sigma(E) e^{-E / k_{B} T},
$$

where $\mu=M_{1} M_{2} /\left(M_{1}+M_{2}\right)$ is the reduced mass of the two collision partners whose masses are $M_{1}$ and $M_{2}, k_{B}$ is the 
Boltzmann constant, and $T$ the environmental temperature. Expressing the cross-section, $\sigma(E)$, in terms of the astrophysical $S$-factor, $S(E)$,

$$
\sigma(E)=\frac{S(E)}{E} e^{-\sqrt{E_{G} / E}},
$$

the reaction rate becomes

$$
\langle\sigma v\rangle=\left(\frac{8}{\pi \mu}\right)^{1 / 2}\left(\frac{1}{k_{B} T}\right)^{3 / 2} \int_{0}^{\infty} d E S(E) e^{-F(E)},
$$

where

$$
F(E)=E / k_{B} T+\sqrt{E_{G} / E},
$$

where

$$
E_{G}=b^{2} \equiv 2 \mu c^{2}\left(\pi Z_{1} Z_{2} \alpha\right)^{2},
$$

$\alpha$ being the fine structure constant. The function $F(E)$ has a single minimum in the interval $[0, \infty)$ from which it increases monotonically to infinity as $E$ approaches zero and infinity. The position of the minimum is given by

$$
E_{0}=\left[E_{G}^{1 / 2} k_{B} T / 2\right]^{2 / 3},
$$

and is the effective energy of the Gamow window for thermonuclear reactions at temperature $T$.

We assume that for low enough energy, $S(E)$ for the radiative capture of a charged particle through a single isolated resonance may be approximated by

$$
S(E)=S_{r} \frac{\Gamma_{r}^{2} / 4}{\left(E-E_{r}\right)^{2}+\Gamma_{r}^{2} / 4},
$$

where $E_{r}$ is the energy of the resonance, $\Gamma_{r}=\Gamma\left(E_{r}\right)$ is it's width at $E_{r}$ and $S_{r}=S\left(E_{r}\right)$. In terms of the resonance strength $(\omega \gamma)_{r}=\sigma\left(E_{r}\right) \mu \Gamma_{r} E_{r} /\left(2 \pi \hbar^{2}\right)$, we have

$$
S_{r}=\frac{2 \pi \hbar^{2}}{\mu} \frac{(\omega \gamma)_{r}}{\Gamma} e^{\sqrt{E_{G} / E_{r}}} .
$$

The reaction rate becomes

$$
\langle\sigma v\rangle=\left(\frac{8}{\pi \mu}\right)^{1 / 2}\left(\frac{1}{k_{B} T}\right)^{3 / 2} S_{r} \Gamma_{r}^{2} / 4 \int_{0}^{\infty} d E \frac{e^{-F(E)}}{\left(E-E_{r}\right)^{2}+\Gamma_{r}^{2} / 4} .
$$

Introducing the dimensionless variables

$$
\begin{gathered}
x=E / k_{B} T, x_{r}=E_{r} / k_{B} T, \xi_{r}=\Gamma_{r} / k_{B} T, \\
x_{G}=a^{2} \equiv E_{G} / k_{B} T,
\end{gathered}
$$

and

$$
\begin{aligned}
x_{0} & =E_{0} / k_{B} T \\
& =\left[x_{G}^{1 / 2} / 2\right]^{2 / 3},
\end{aligned}
$$

one can rewrite the reaction rate as

$$
\langle\sigma v\rangle=\left(\frac{8}{\pi \mu}\right)^{1 / 2}\left(\frac{1}{k_{B} T}\right)^{5 / 2} S_{r} \frac{\Gamma_{r}^{2}}{4} I\left(x_{0}, x_{p}\right)
$$

where

$$
I\left(x_{0}, x_{p}\right)=\int_{0}^{\infty} d x \frac{e^{-f(x)}}{\left(x-x_{r}\right)^{2}+\xi_{r}^{2} / 4}
$$

$$
=\frac{2}{\xi_{r}} \mathfrak{J} J\left(x_{0}, x_{p}\right),
$$

$$
J\left(x_{0}, x_{p}\right)=\int_{0}^{\infty} d x \frac{e^{-f(x)}}{x-x_{p}},
$$

$$
x_{p} \equiv x_{r}+i \xi_{r} / 2 \text {, }
$$

and

$$
\begin{aligned}
f(x) & \equiv F\left(k_{B} T x\right) \\
& =x+\sqrt{x_{G} / x} .
\end{aligned}
$$

We have given $I$ and $J$ the arguments $x_{0}$ and $x_{p}$, the minimum of $f(x)$ and the position of the pole due to the resonance, respectively. These are the critical points $[6,12]$ of the integrand. The principal contributions to an integral such as we are considering come from the neighborhood of the critical points.

\section{CONVENTIONAL APPROXIMATIONS}

When the resonance and the Gamow window are well separated the contribution from each to the reaction rate can be estimated separately and the two contributions summed. Let us write

$$
I \approx I_{r}+I_{G}
$$

where $I_{r}$ is the contribution from the resonance and $I_{G}$ the contribution from the Gamow window. The resonance contribution may be estimated [11] by approximating the Gamow window exponential in Eq. (14) by its value at the resonance energy, $x_{r}$ :

$$
I_{r}=e^{-f\left(x_{r}\right)} \int_{0}^{\infty} \frac{d x}{\left(x-x_{r}\right)^{2}+\xi_{r}^{2} / 4}=\frac{e^{-x_{r}-\sqrt{x_{G} / x_{r}}}}{\xi_{r} / 2}\left(\pi / 2+\theta_{r}\right),
$$

with

$$
\theta_{r}=\tan ^{-1}\left(2 x_{r} / \xi_{r}\right) .
$$

Similarly, the Gamow window contribution is estimated by approximating the Breit-Wigner factor by its value at the location of the Gamow peak, $x_{0}$ :

$$
\begin{aligned}
I_{G} & =\frac{e^{-f\left(x_{0}\right)}}{\left(x_{0}-x_{r}\right)^{2}+\xi_{r}^{2} / 4} \int_{0}^{\infty} d x e^{-\left[f(x)-f\left(x_{0}\right)\right]} \\
& \approx \frac{e^{-f\left(x_{0}\right)}}{\left(x_{0}-x_{r}\right)^{2}+\xi_{r}^{2} / 4} \int_{0}^{\infty} d x e^{-\left(x-x_{0}\right)^{2} /\left(\Delta_{x} / 2\right)^{2}}
\end{aligned}
$$



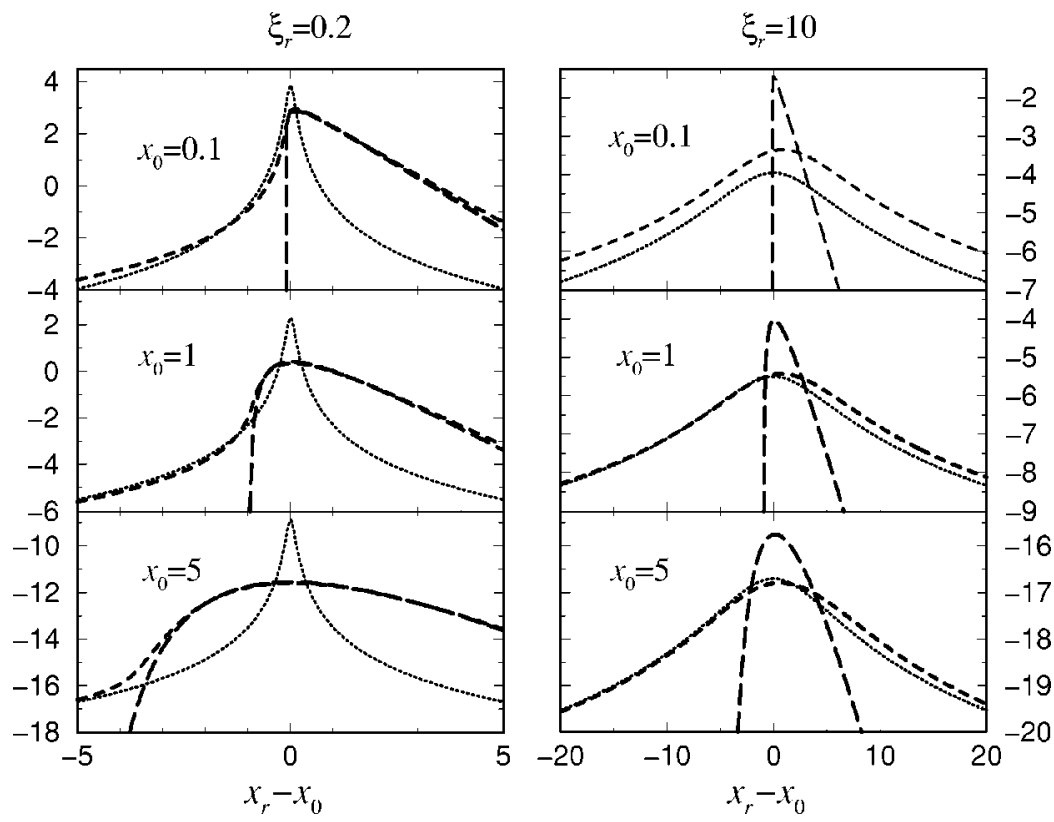

In Eq. (24) a Gaussian approximation to the Gamow window exponential has been employed [11] and we have introduced

$$
\Delta_{x}=\sqrt{8 / F^{\prime \prime}\left(x_{0}\right)}=4 \sqrt{x_{0} / 3},
$$

the effective width of the Gamow window in units of $k_{B} T$ and

$$
\tau=f\left(x_{0}\right)=3 x_{0} .
$$

Equation (24) is the zeroth-order term of an asymptotic expansion of $I_{G}$ in powers of $1 / \tau$ [5]. For the reaction rate one thus obtains

$$
\begin{aligned}
\langle\sigma v\rangle \approx & (2 / \mu)^{1 / 2} \frac{1}{\left(k_{B} T\right)^{3 / 2}}\left[\pi^{1 / 2} \Gamma S\left(E_{r}\right) e^{-E_{r} / k_{B} T-\sqrt{E_{G} / E_{r}}}\right. \\
& \left.+\Delta S\left(E_{0}\right) e^{-\tau}\right]
\end{aligned}
$$

where $\Delta=4\left(E_{0} k_{B} T / 3\right)^{1 / 2}$ is the width of the Gamow window.

In Fig. 1 we plot $I_{r}, I_{G}$ and $I_{r}+I_{G}$ together with the exact $I$ for comparison over a range of parameters. The left- and right-hand columns are representative of narrow resonances, $\xi_{r} / 2=0.1$, and wide resonances, $\xi_{r} / 2=5.0$, respectively. The three rows show the evolution with respect to $a$, which determines both the effective energy $x_{0}$ and effective width of the Gamow window $\Delta_{x}$.

It is seen from Fig. 1 that when $x_{r} \sim x_{0}$ the method of approximating $I$ by $I_{r}+I_{G}$ fails for both narrow and wide resonances: for narrow resonances, $I_{G}$ alone overestimates $I$ whilst for wide resonances $I_{r}$ alone overestimates $I$.

\section{UNIFORM APPROXIMATION}

We now introduce new variable $t$ implicitly by

$$
f(x)-f\left(x_{0}\right)=t^{2} .
$$

This transformation satisfies

FIG. 1. A comparison of exact reaction rate with conventional approximations. The dashed lines show $\log _{10} I$ [Eq. (14)], the long dashed lines $\log _{10} I_{r}$ [Eq. (21)], the dotted lines $\log _{10} I_{G}$ [Eq. (24)] vs resonance energy (shifted by the effective energy). See also Table I.

$$
\begin{gathered}
t(x=0)=-\infty, t(x=\infty)=\infty, \\
t\left(x=x_{p}\right)=t_{p}, t\left(x=x_{0}\right)=0 .
\end{gathered}
$$

The position of the pole in $t$-space is then given by

$$
\begin{aligned}
t_{p} & =\left[f\left(x_{p}\right)-f\left(x_{0}\right)\right]^{1 / 2} \\
& =\left[\frac{E_{r}-i \Gamma_{r} / 2-E_{0}}{k_{B} T}+\sqrt{\frac{E_{G}}{E_{r}-i \Gamma_{r} / 2}}-\sqrt{\frac{E_{G}}{E_{0}}}\right]^{1 / 2} .
\end{aligned}
$$

Equation (15) becomes

$$
\begin{aligned}
J\left(x_{0}, x_{p}\right) & =\int_{-\infty}^{\infty} d t \frac{d x}{d t} \frac{t-t_{p}}{x-x_{p}} \frac{e^{-t^{2}-\tau}}{t-t_{p}} \\
& =e^{-\tau} \int_{-\infty}^{\infty} d t \Phi(t) \frac{e^{-t^{2}}}{t-t_{p}},
\end{aligned}
$$

where

$$
\Phi(t)=\frac{d x}{d t} \frac{t-t_{p}}{x-x_{p}} .
$$

In order to obtain an expansion which is uniform as $x_{p}$ is made to approach or coincide with $x_{0}, \Phi(t)$ is expanded as follows [12-14]:

$$
\Phi(t)=\sum_{n=0}^{\infty}\left[\alpha_{n}+\left(t-t_{p}\right) \beta_{n}\right]\left[t\left(t-t_{p}\right)\right]^{n} .
$$

The expansion given by Eq. (34) has the property that it gives the value of the function $\Phi(t)$ and all its derivatives exactly at both $t=0$ and $t=t_{p}$. Let us approximate $\Phi(t)$ by the truncation

$$
\Phi_{0}(t)=\alpha_{0}+\left(t-t_{p}\right) \beta_{0},
$$

Then $J\left(x_{0}, x_{p}\right)$ is approximated by 


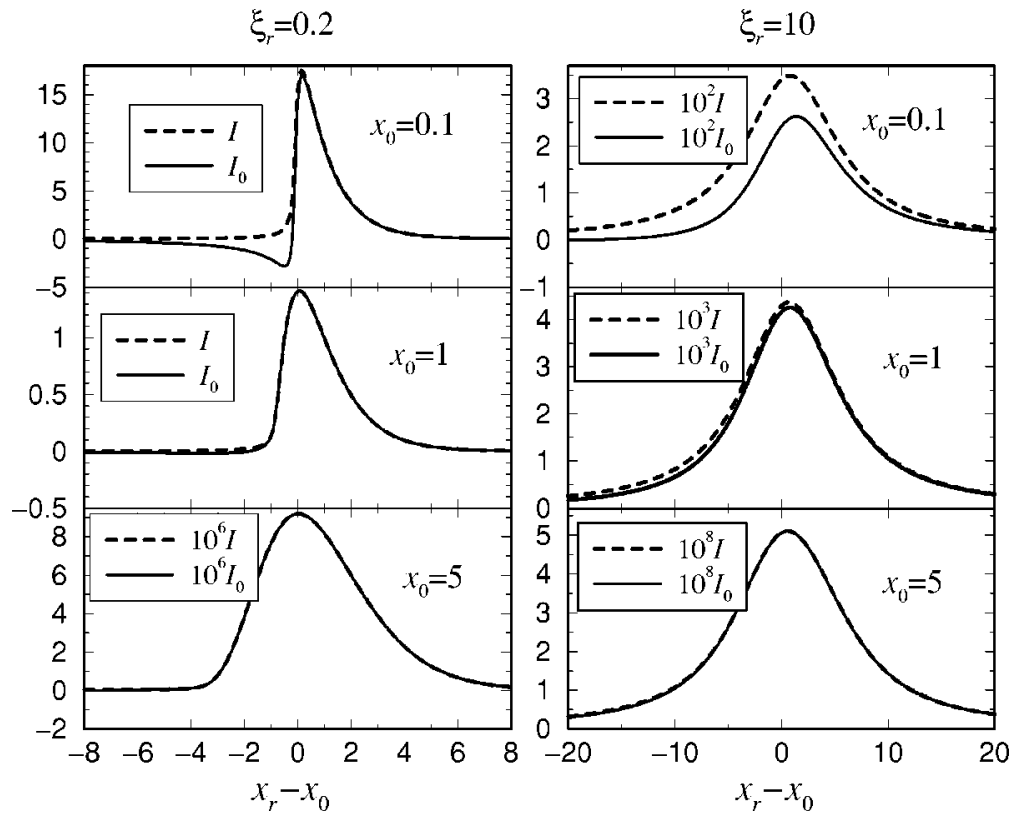

FIG. 2. The dashed lines show $I$ [Eq. (14)] and the solid lines the uniform approximation $I_{0}$ [Eq. (40)] vs resonance energy (shifted by the effective energy). See also Table I.

$$
\begin{aligned}
J_{0}\left(x_{0}, x_{p}\right) & =e^{-\tau} \int_{-\infty}^{\infty} d t \Phi_{0}(t) \frac{e^{-t^{2}}}{t-t_{p}} \\
& =\alpha_{0} e^{-\tau} \int_{-\infty}^{\infty} d t \frac{e^{-t^{2}}}{t-t_{p}}+\beta_{0} e^{-\tau} \int_{-\infty}^{\infty} d t e^{-t^{2}} \\
& =\alpha_{0} e^{-\tau} \int_{-\infty}^{\infty} d t \frac{e^{-t^{2}}}{t-t_{p}}+\beta_{0} \sqrt{\pi} e^{-\tau} .
\end{aligned}
$$

In the Appendix it is shown that $\alpha_{0}=1$ and

$$
\beta_{0}=\frac{1}{t_{p}}+\frac{2 \sqrt{\tau}}{3\left(x_{0}-x_{p}\right)} .
$$

Introducing the complementary error function through the relation [15],

$$
\int_{-\infty}^{\infty} d t \frac{e^{-t^{2}}}{t-z}=i \pi e^{-z^{2}} \operatorname{erfc}(-i z)
$$

we obtain an approximation to $I\left(x_{0}, x_{p}\right)$ which corresponds to the truncation of the Taylor series we made in Eq. (35):

$$
I_{0}=I_{\alpha_{0}}+I_{\beta_{0}}
$$

where

$$
I_{\alpha_{0}}=\frac{\pi}{\xi_{r} / 2} e^{-\tau} \mathfrak{J} i e^{-t_{p}^{2}} \operatorname{erfc}\left(-i t_{p}\right)
$$

and

$$
I_{\beta_{0}}=I_{\beta_{0}}^{(p)}+I_{\beta_{0}}^{(q)},
$$

where

$$
I_{\beta_{0}}^{(p)}=\frac{\sqrt{\pi}}{\xi_{r} / 2} e^{-\tau} \mathfrak{J} \frac{1}{t_{p}},
$$

where $I_{G}$ is given by Eq. (24). Substituting Eqs. (40)-(44) into Eq. (13) we finally obtain

$$
\begin{aligned}
\langle\sigma v\rangle= & (2 / \mu)^{1 / 2} \frac{e^{-\tau}}{\left(k_{B} T\right)^{3 / 2}}\left[\Gamma S\left(E_{r}\right) \mathfrak{J}\left\{i \sqrt{\pi} e^{-t_{p}^{2}} \operatorname{erfc}\left(-i t_{p}\right)+\frac{1}{t_{p}}\right\}\right. \\
& \left.+\Delta S\left(E_{0}\right)\right]
\end{aligned}
$$

where we have expressed our result using the astrophysical $S$-factor, $S(E)=\sigma(E) E e^{\sqrt{E_{G} / E}}$ and the width of the Gamow window $\Delta=4\left(E_{0} k_{B} T / 3\right)^{1 / 2}$. In Fig. 2 we compare $I$ with the uniform approximation $I_{0}$. We see that $I_{0}$ gives a good account of $I$ for the whole of the parameter range investigated (Table I).

In order to understand the contribution of the three terms on the r.h.s. of Eq. (45) let us consider Eq. (30) for $t_{p}$. Writing $x_{p}=x_{0}+\delta x$ where $\delta x=x_{p}-x_{0}$ we obtain

$$
\begin{aligned}
t_{p} & =\left[x_{p}+\sqrt{x_{G} / x_{p}}-3 x_{0}\right]^{1 / 2} \\
& =\left[x_{0}+\delta x+\sqrt{x_{G} / x_{0}}\left(1+\delta x / x_{0}\right)^{-1 / 2}-3 x_{0}\right]^{1 / 2} .
\end{aligned}
$$

Noting that $\sqrt{x_{G} / x_{0}}=2 x_{0}$ and making the binomial expansion

TABLE I. Parameter values used in Figs. 1-3.

\begin{tabular}{cccc}
\hline \hline$x_{0}$ & 0.1 & 1.0 & 5.0 \\
\hline$\tau$ & 0.3 & 3.0 & 15.0 \\
$a$ & 0.063 & 2.0 & 22.36 \\
$x_{G}$ & 0.004 & 4.0 & 500.0 \\
$\Delta_{x}$ & 0.73 & 2.31 & 5.16 \\
\hline \hline
\end{tabular}




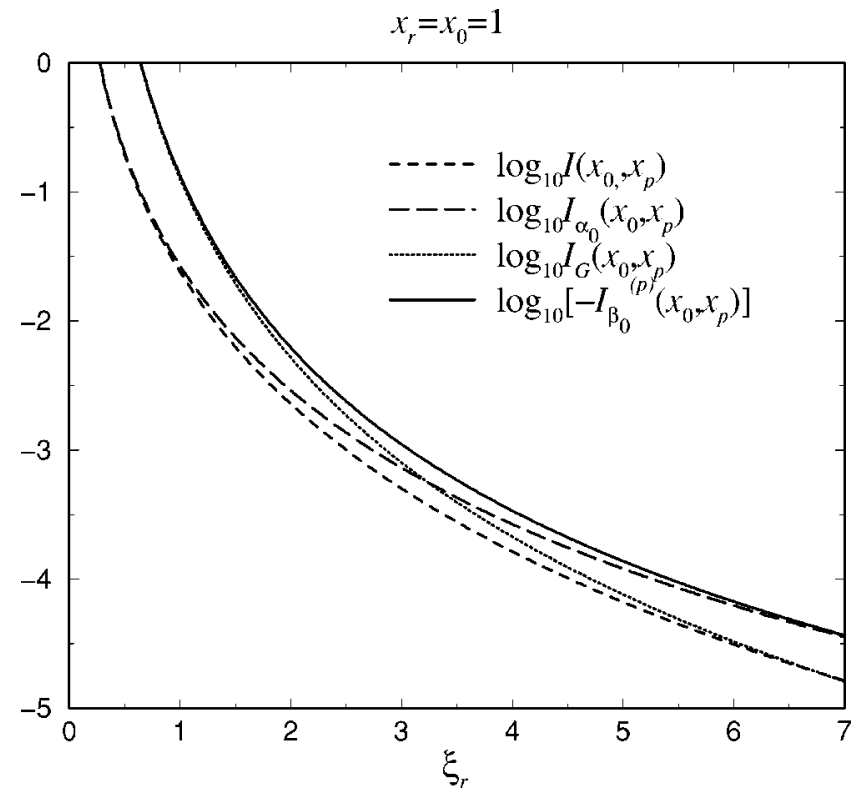

FIG. 3. Comparison of the three terms of the uniform approximation with the exact $I$ (dashed line) [Eq. (14)] as a function of $\xi_{r}=\Gamma_{r} / k_{B} T$. The long dashed lines show $\log _{10} I_{\alpha_{0}}$ [Eq. (41)], the solid lines $\log _{10}\left[-I_{\beta_{0}}^{(p)}\right]$ [Eq. (43)] and the dotted lines $\log _{10} I_{G}[$ Eq. (24)].

$$
\begin{aligned}
\left(1+\delta x / x_{0}\right)^{-1 / 2} & =\sum_{j=0}^{\infty}\left(\begin{array}{c}
-1 / 2 \\
j
\end{array}\right) \delta x^{j} / x_{0}^{j} \\
& =1-\frac{1}{2} \frac{\delta x}{x_{0}}+\frac{3}{8} \frac{\delta x^{2}}{x_{0}^{2}}-\frac{5}{16} \frac{\delta x^{3}}{x_{0}^{3}}+\cdots
\end{aligned}
$$

we obtain

$$
\begin{aligned}
t_{p}^{-1} & =\frac{1}{\sqrt{2 x_{0}}}\left[\frac{3}{8} \frac{\delta x^{2}}{x_{0}^{2}}-\frac{5}{16} \frac{\delta x^{3}}{x_{0}^{3}}+\cdots\right]^{-1 / 2} \\
& =\frac{2 \sqrt{x_{0} / 3}}{\delta x}\left[\frac{8}{3} \sum_{j=0}^{\infty}\left(\begin{array}{c}
-1 / 2 \\
j+2
\end{array}\right) \delta x^{j} / x_{0}^{j}\right]^{-1 / 2} .
\end{aligned}
$$

In the limit that $\delta x \rightarrow 0$ the series in Eq. (47) may be approximated by

$$
\begin{aligned}
t_{p} & \approx \frac{x_{p}-x_{0}}{2 \sqrt{x_{0} / 3}} \\
& =\frac{E_{r}-E_{0}-i \Gamma_{r} / 2}{\Delta / 2} .
\end{aligned}
$$

Substituting this expression for $t_{p}$ into Eq. (43) we see that $I_{\beta_{0}}^{(p)}$ cancels $I_{\beta_{0}}^{(q)}$ so that $I \approx I_{\alpha_{0}}$. This behavior is confirmed numerically by Fig. 3 for $x_{r}=x_{0}$. The result $I \approx I_{r}$ [Eq. (21)] may be recovered by making the crude approximation that $\operatorname{erfc}\left(-i t_{p}\right) \sim 1$ for $t_{p} \sim 0$ and assuming that $\Gamma_{r} \gg E_{r}$.

To analyze the limit $\delta x \rightarrow \infty$ we write $-i t_{p}=z$ and employ the asymptotic series [15]

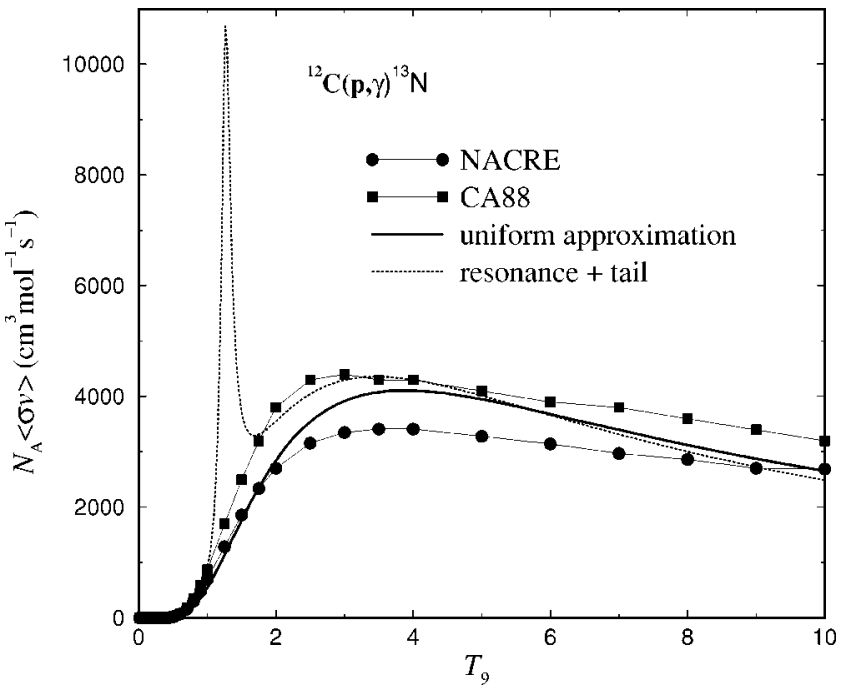

FIG. 4. The reaction rate for ${ }^{12} \mathrm{C}(p, \gamma){ }^{13} \mathrm{~N}$ as function of $T_{9}$. The circles show the rates from Ref. [1], the squares Ref. [17], the solid lines the uniform approximation, Eq. (45), and the dotted lines the resonance-plus-tail approximation, Eq. (27). We took the resonance parameters from Ref. [16] $\left(E_{r}=457 \mathrm{KeV}\right.$ and $\Gamma_{r} \approx \Gamma_{p}\left(E_{r}\right)$ $=39 \mathrm{KeV}$ [both being laboratory frame values] and $\sigma\left(E_{r}\right)=130 \mu$ b).

$$
\sqrt{\pi} e^{z^{2}} \operatorname{erfc}(z)=\frac{1}{z}-\frac{1}{2 z^{3}}+\frac{3}{4 x^{5}}-\frac{15}{8 x^{7}}+\cdots,
$$

which is valid for $z \rightarrow \infty$. This allows us to see that

$$
i \sqrt{\pi} e^{-t_{p}^{2}} \operatorname{erfc}\left(-i t_{p}\right)+\frac{1}{t_{p}}=\frac{1}{2 t_{p}^{3}}-\frac{3}{4 t_{p}^{5}}+\frac{15}{8 t_{p}^{7}}-\cdots
$$

is $O\left(1 / \delta x^{3}\right)$. Thus $I \approx I_{G}$ as $\delta x \rightarrow \infty$ since $I_{G} \sim O(1 / \delta x)$. This behavior is also confirmed by Fig. 3 for $x_{r}=x_{0}$. When $x_{r}$ $-x_{0} \gg 0$ the conventional approximation [Eq. (20)] may be used.

\section{REACTION RATE FOR ${ }^{12} \mathrm{C}(p, \gamma){ }^{13} \mathrm{~N}$}

We have calculated the reaction rate for ${ }^{12} \mathrm{C}(p, \gamma){ }^{13} \mathrm{~N}$ which is important in the CNO cycle using Eqs. (45) and (27) including only the lowest resonance at $457 \mathrm{keV}$ [16] and the results are displayed in Fig. 4. The exact integral is not shown as it is indistinguishable from the uniform approximation for this case. It is apparent that the resonanceplus-tail approximation cannot be used at temperatures for which $E_{r} \sim E_{0}$. For comparison we have also shown the rates of Ref. [17] which used a different analytic approach and those of Ref. [1] which also included the contribution of the second resonance at $1698 \mathrm{keV}$.

\section{CONCLUSIONS}

In this paper we have derived a new approximate analytic expression for the thermonuclear reaction rate when the cross section has a single isolated resonance. The new expression has a much wider range of validity than simpler approximations which are commonly used. In particular it is uniformly 
valid as the resonance energy coalesces with the effective energy of the Gamow window and as the width of the resonance is varied from narrow to broad. An interesting problem which still has to be addressed is the generalization to several resonances.

\section{ACKNOWLEDGMENTS}

This work was supported by FAPESP and CNPq.

\section{APPENDIX: CALCULATION OF $\alpha_{0}$ AND $\beta_{0}$}

The coefficients $\alpha_{0}$ and $\beta_{0}$ are obtained from Eq. (35) together with Eq. (29). One finds

$$
\begin{aligned}
\alpha_{0} & =\Phi_{0}\left(t_{p}\right) \\
& =\left.\frac{d x}{d t}\right|_{t=t_{p}}\left[\frac{t-t_{p}}{x-x_{p}}\right]_{t=t_{p}} \\
& =\left.\left.\frac{d x}{d t}\right|_{t=t_{p}} \cdot \frac{d t}{d x}\right|_{t=t_{p}} \\
& =1
\end{aligned}
$$

and

$$
\beta_{0}=\frac{\Phi_{0}\left(t_{p}\right)-\Phi_{0}(0)}{t_{p}}=\frac{1}{t_{p}}+\left.\frac{d x}{d t}\right|_{t=0} \frac{1}{x_{0}-x_{p}} .
$$

Here, $\quad d x /\left.d t\right|_{t=0}$ is obtained from

$$
\begin{gathered}
\frac{d f(x)}{d t}=f^{\prime}(x) \frac{d x}{d t}=2 t \\
\frac{d^{2} f(x)}{d t^{2}}=f^{\prime \prime}(x)\left(\frac{d x}{d t}\right)^{2}+f^{\prime}(x) \frac{d^{2} x}{d t^{2}}=2 .
\end{gathered}
$$

Upon using

$$
\begin{gathered}
f^{\prime}\left(x_{0}\right)=0, \\
f^{\prime \prime}\left(x_{0}\right)=\frac{9}{2 \tau},
\end{gathered}
$$

we find

$$
\left.\frac{d x}{d t}\right|_{t=0}=\sqrt{2 / f^{\prime \prime}\left(x_{0}\right)}=\frac{2}{3} \sqrt{\tau},
$$

and thus $\beta_{0}$ is given by Eq. (38).
[1] C. Angulo, M. Arnould, M. Rayet, P. Descouvemont, D. Baye, C. Leclercq-Willain, A. Coc, S. Barhoumi, P. Aguer, C. Rolfs et al., Nucl. Phys. A656, 3 (1999).

[2] C. Iliadis, J. M. D'Auria, S. Starrfield, W. J. Thompson, and M. Wiescher, Astrophys. J., Suppl. Ser. 134, 151 (2001).

[3] H. Herndl, R. Hofinger, J. Jank, H. Oberhummer, J. Görres, M. Wiescher, F.-K. Thielemann, and B. A. Brown, Phys. Rev. C 60, 064614 (1999).

[4] A. M. Mathai and H. J. Haubold, Astrophys. Space Sci. 282, 265 (2002).

[5] M. Ueda, A. J. Sargeant, M. P. Pato, and M. S. Hussein, Phys. Rev. C 61, 045801 (2000).

[6] R. B. Dingle, Asymptotic Expansions: Their Derivation and Interpretation (Academic, New York, 1973).

[7] E. G. Adelberger, S. M. Austin, J. N. Bahcall, A. B. Balantekin, G. Bogaert, L. S. Brown, L. Buchmann, F. E. Cecil, A. E. Champagne, L. de Braeckeleer et al., Rev. Mod. Phys. 70, 1265 (1998).
[8] B. K. Jennings, S. Karataglidis, and T. D. Shoppa, Phys. Rev. C 58, 3711 (1998).

[9] A. M. Mukhamedzhanov and F. M. Nunes, Nucl. Phys. A708, 437 (2002).

[10] S. Goriely, Phys. Lett. B 436, 10 (1998).

[11] C. E. Rolfs and W. S. Rodney, Cauldrons in the Cosmos (Chicago Univ. Press, Chicago, 1988).

[12] N. Bleistein and R. A. Handelsman, Asymptotic Expansions of Integrals (Holt, Rinehart and Winston, New York, 1986) (reprinted in 1986 by Dover, New York).

[13] C. Chester, B. Friedman, and F. Ursell, Proc. Cambridge Philos. Soc. 53, 599 (1957).

[14] M. V. Berry, Proc. Phys. Soc. London 89, 479 (1966).

[15] Handbook of Mathematical Functions, edited by M. Abramowitz and I. A. Stegun (Dover, New York, 1970).

[16] C. Rolfs and R. E. Azuma, Nucl. Phys. A227, 291 (1974).

[17] G. R. Caughlan and W. A. Fowler, At. Data Nucl. Data Tables 40, 283 (1988). 